Check for updates

Cite this: RSC Adv., 2019, 9, 28330

Received 24th June 2019

Accepted 23rd August 2019

DOI: $10.1039 / c 9 r a 04761 a$

rsc.li/rsc-advances

\title{
Formation of concentrated triglyceride nanoemulsions and nanogels: natural emulsifiers and high power ultrasound
}

\author{
Mohammad Nejatian and Soleiman Abbasi iD *
}

\begin{abstract}
The fabrication of concentrated nanoemulsions provides potential advantages such as loading capacity enhancement, storage and transportation costs reduction, and creation of novel textures. The current study investigated the capability of high power ultrasound on nanoemulsification of high concentration triglyceride using various natural emulsifiers (saponin, whey protein isolate, lecithin and sucrose monopalmitate). The impact of the emulsifier concentration (up to $6 \mathrm{wt} \%$ ), oil content (up to $60 \mathrm{wt} \%$ ) and exposure to sonication (up to $33 \mathrm{~min}$ ) on the droplet size distribution, physical stability and rheological properties were evaluated. Regarding the dilute nanoemulsion (10 wt\% oil), droplet size was inversely correlated with the concentration of emulsifiers, however only by using saponin (2 wt\%) the droplet size was in nano range $(d<200 \mathrm{~nm})$. The concentrated nanoemulsions $(20-50 \mathrm{wt} \%)$ were also fabricated under sonication (15 min at saponin-to-oil ratio $2: 10 \mathrm{w} / \mathrm{w} \%$ ). They also presented shearthinning behavior with relatively low consistency coefficients. Surprisingly, the one with $60 \mathrm{wt} \%$ oil was easily converted to viscoelastic gel upon $3 \mathrm{~min}$ sonication. Owing to such characteristics, they could have potential applicability in formulation of soft foods, creams, sauces, salad dressings, pastes, lotions, cosmetics and pharmaceuticals.
\end{abstract}

\section{Introduction}

The formation of nanoemulsions is one of the interesting applications of nanotechnology in foods, supplements, personal-care products, cosmetics, pharmaceuticals and many other industries. They usually act as carriers for controlled delivery of antimicrobials and health promoting compounds namely; vitamins, nutraceuticals, flavoring and coloring agents. ${ }^{1-3}$ Moreover, they could prolong shelf life owing to their high kinetic stability against various destabilizing phenomena. These advantages mainly arise from their small droplet size $(d<$ $200 \mathrm{~nm}$ ) which dramatically increase their contact surface with the continuous aqueous phase., ${ }^{4,5}$

However, most nanoemulsions to date are diluted, but in certain applications, particularly in foods, cosmetics and pharmaceuticals, it is worthwhile to prepare concentrated nanoemulsions. For example, concentrated nanoemulsions and nanogels could potentially improve the loading capacity of liphophilic ingredients in nano-encapsulation systems for gels, creams, and pastes. ${ }^{6}$ Furthermore, due to lower storage and transportation costs, they have immediate economic advantages, because they can be simply diluted at the formulation

Food Colloids and Rheology Lab., Department of Food Science \& Technology, Faculty of Agriculture, Tarbiat Modares University, P O Box 14115-336, Tehran, Iran. E-mail: sabbasifood@modares.ac.ir; Fax: $\quad$ +982144196524; $\quad$ +982148292200; $\quad$ Tel: $+982148292321$ sites. $^{7}$ They can also turn to highly viscous or gel-like structures at much lower dispersed phase volume fractions than conventional emulsions. Such advantages could be useful in the production of low fat foods, with a consistency similar to original one, without using fat substitutes. ${ }^{\mathbf{8}, 9}$

The nature and concentration of emulsifiers, homogenization method as well as processing conditions are usually the most influencing parameters of the concentrated nanoemulsion production. ${ }^{\mathbf{1 0 , 1 1}}$ In practice, the production of such nanomulsions seems to be more difficult, because the collision frequency of oil droplets and consequently their coalescence are proportional to the concentration of droplets, the high the oil content the more the collisions. Based on a most recent report, the concentrated nanoemulsions and nanogels can be fabricated by using high power ultrasound using a synthetic surfactant. ${ }^{\mathbf{1 2}}$ However, due to their potential health concerns, the present study attempted to evaluate the possibility of fabricating the concentrated triglyceride (sunflower oil) nanoemulsions using natural emulsifiers.

The application of ultrasonic homogenization, using a wide variety of surfactants and co-surfactants, has been successfully examined in production of nanoemulsions from diverse lipid phase namely long-chain triglycerides (LCT), ${ }^{\mathbf{1 3 - 1 5}}$ medium-chain triglycerides or MCT, ${ }^{16}$ essential oils ${ }^{17-19}$ and lipophilic nutraceuticals. $^{2021}$ However, it has rarely, if none, been used to produce concentrated LCT nanoemulsions using natural emulsifiers. In addition, the employed technique has some 
advantages namely; less energy consumption, low capital investment and maintenance costs, ease of operation, and low equipment contamination over other high energy methods. ${ }^{22}$ The high power ultrasound generates nanoemulsions through propagating the high-intensity (low frequency) ultrasonic waves into the dispersion. They produce alternating high- (compression) and low-pressure (rarefaction) cycles during which air bubbles form, grow and eventually, at a critical volume, collapse. Such a violent collapse generates high shear forces, intense local heating and turbulence which results in droplet disruption in nanometer dimensions. ${ }^{23}$

Selecting an appropriate emulsifier is the most challenging part of nano-emulsification process. The safety and effectiveness of the selected emulsifiers are also highly concerned particularly in edible products. An ideal emulsifier should have rapid adsorption rate on the freshly formed interface during homogenization, high capability to reduce the interfacial tension and preventing the droplet aggregation. ${ }^{\mathbf{1 1}}$ There are a number of synthetic food grade surfactants with these characteristics but most of them are potentially irritant and even toxic particularly at high consumption rates. ${ }^{24}$ These facts along with growing public awareness have increased the consumer and industry demands on natural and bio-based ingredients. Accordingly, during the last two decades scientists attempted to examine the ability of proteins,${ }^{25-27}$ polysaccharides, ${ }^{28,29}$ phospholipids $^{30}$ and small molecule bio-based emulsifiers ${ }^{31}$ on the formation and the stabilization of edible emulsions.

The current study was therefore aimed to determine whether the concentrated nanoemulsions could be formed under high power ultrasonication by using commercially available natural emulsifiers (saponin, whey protein isolate or WPI, lecithin and sucrose monopalmitate or SMP). The influence of different sonication conditions as well as the emulsifier concentration on the formation and stability of the nanoemulsions was also examined. Finally, the rheological properties of the resulted nanoemulsions were determined. The rheological findings could provide invaluable information regarding their applicability in formulations, designing and predicting flow behavior

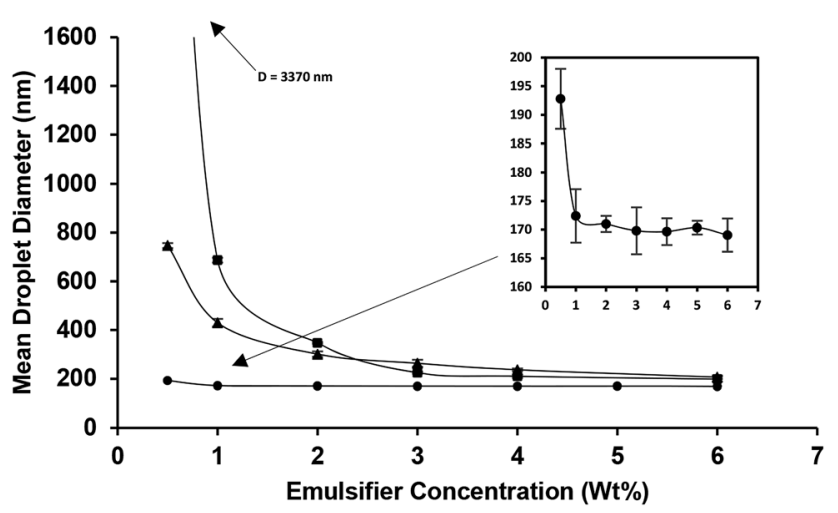

Fig. 1 Influence of emulsifier type [saponin (-), WPI ( $\mathbf{\Delta})$ and lecithin (曾)] and concentration on the mean droplet diameter of $10 \mathrm{wt} \%$ oilin-water (nano)emulsions prepared under pulsed sonication $(9 \mathrm{~min}$, $100 \%$ amplitude). The inset magnifies the mean droplet diameter changes of nanoemulsions prepared by using saponin. in different unit operations (mixing, transportation, filling and pasteurization) and even oral food processing.

\section{Materials and methods}

\subsection{Materials}

Refined sunflower oil (NINA®), as an example of a LCT, was purchased from local grocery shop and stored at room temperature in dark. Saponin (pharmaceutical grade) was provided by Pioneer Biotech Co. (Shaanxi, China). Whey protein isolate or WPI (GermanProt 9000, protein content $92.16 \%$ in dry matter) was purchased from Sachsenmilch Leppersdorf GmbH (Leppersdorf, Germany). Lecithin (Yelkin ${ }^{\mathrm{TM}}$ ) and SMP were obtained from Archer Daniels Midland Company (Decatur, IL, USA) and Compass Foods Company (Tuas, Singapore), respectively. In addition, sodium azide $\left(\mathrm{NaN}_{3}\right)$ and sodium phosphate monobasic/dibasic were supplied by Merck Chemicals Co. (Darmstadt, Germany). For preparation of all solutions deionized water $(18.2 \mathrm{M} \Omega \mathrm{cm})$ was used.

\subsection{Nanoemulsion preparation}

The pre-emulsions $(50 \mathrm{~mL})$ were prepared by mixing the aqueous and oil phases at 9:1 weight ratios. The aqueous phases were formulated by dissolving saponin, WPI and/or SMP powder (0.5-6 wt\%) in a buffer solution (50 $\mathrm{mM}$ phosphate buffer, pH 7.0 containing $0.01 \mathrm{wt} \% \mathrm{NaN}_{3}$, as an antibacterial agent) and stirred for $2 \mathrm{~h}$ to ensure complete dissolution. In case of acidic $\mathrm{pH}$, it was adjusted using $\mathrm{NaOH}$ and $\mathrm{HCl}$ solutions (0.1 M) prior to emulsification. Regarding lecithin, due to its lipophilic nature it was dispersed in oil phase.

The aqueous and oil phases were initially pre-homogenized with a rotor-stator (Wise Tis, Daihan Scientific Co., South Korea) at a speed of $20000 \mathrm{rpm}$ for $1 \mathrm{~min}$. The coarse emulsions were then sonicated (Sonicator 4000, maximum nominal power $600 \mathrm{~W}, 20 \mathrm{kHz}$, Misonix Inc, New York, USA) using a high gain cylindrical titanium sonotrode (dia $=19.1 \mathrm{~mm}$ ) under controlled temperature $\left(30 \pm 5^{\circ} \mathrm{C}\right)$. The amplitude of oscillation was set at $100 \%$ and using a pulsed mode ( $30 \mathrm{~s} \mathrm{ON,} 30 \mathrm{~s}$ OFF) the sonication was lasted up to $33 \mathrm{~min}$. Under sonication conditions, the surfactant-to-oil ratio (SOR) was also optimized. The concentrated nanoemulsions were formulated by premixing higher weight fractions of oil $(20,30,40,50$, and $60 \mathrm{wt} \%)$ with appropriate amount of aqueous phase $(80,70,60,50$, and $40 \mathrm{wt} \%)$ at a constant SOR $(2: 10)$ and emulsified as previously described.

\subsection{Determination of droplet size and $\zeta$ potential}

The mean droplet diameter ( $Z$-average) and size distribution of nanoemulsions were determined by dynamic light scattering (Zetasizer Nano-ZS, Malvern Instruments Ltd., Worcestershire, UK). Prior to analysis, the samples were diluted $(1: 400)$ with buffer solution to avoid multiple scattering effects and obscuration. The relative refractive index (the dispersed $v s$. continuous phases) was 1.455 . The surface potential ( $\zeta$-potential) of the nanoemulsion droplets was also measured using the same apparatus. 
a)

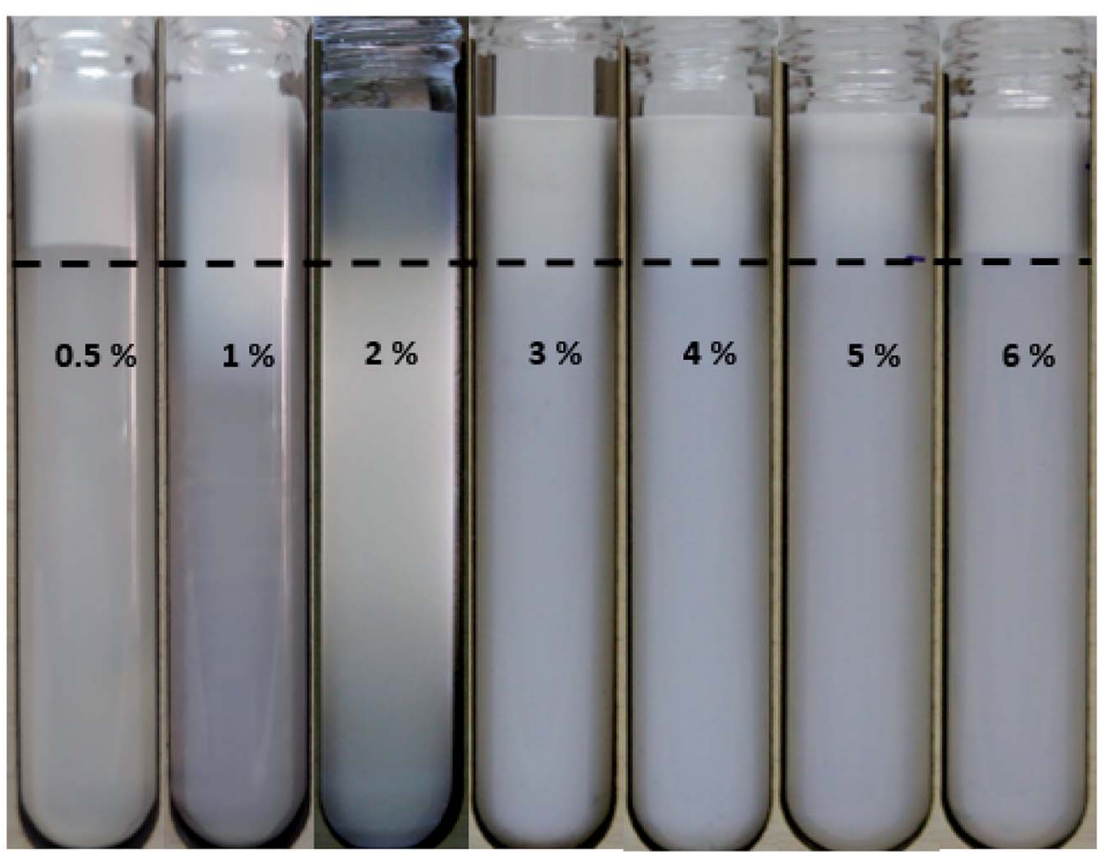

b)

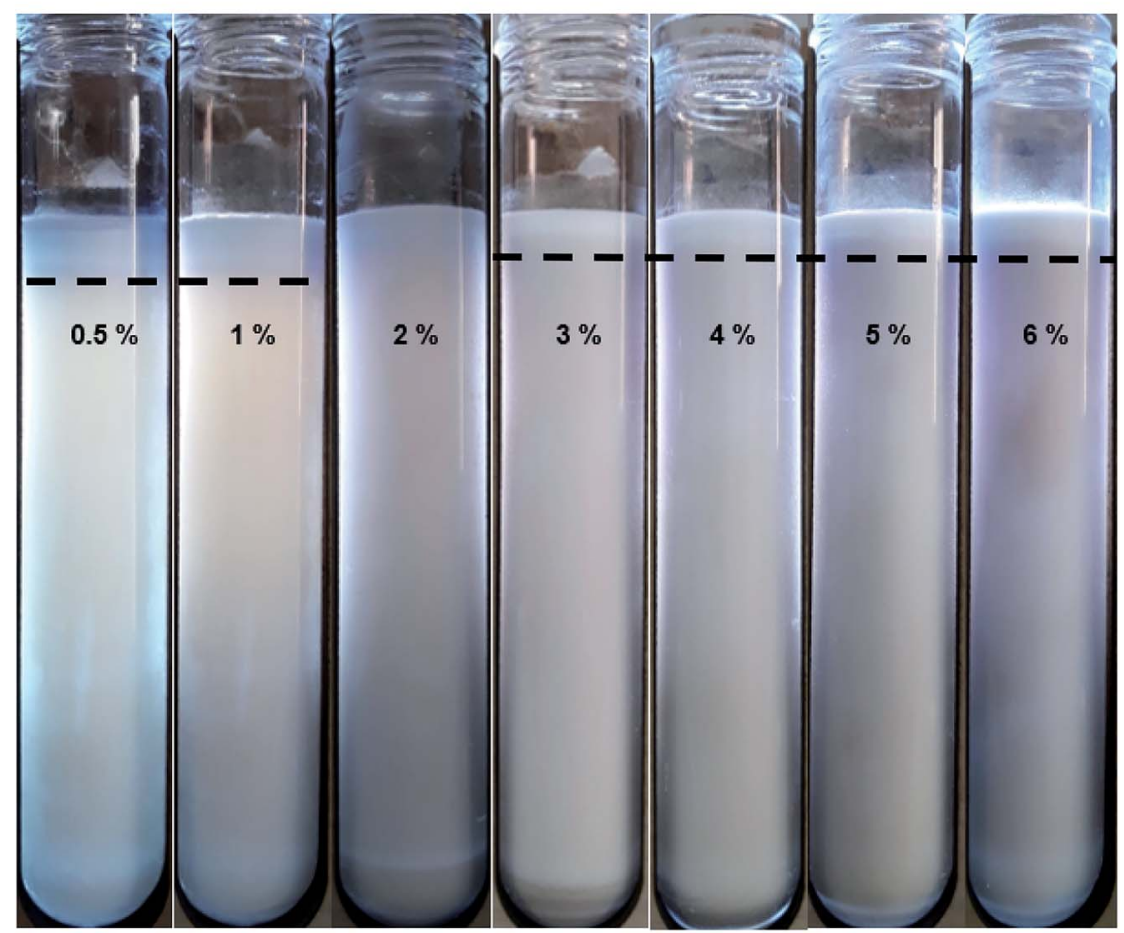

Fig. 2 Influence of various concentration (0.5 to 6 wt\% left to right) of SMP (a) and saponin (b) on the visual appearance of freshly prepared (nano) emulsions (10 wt\% oil) homogenized by ultrasonicator for 9 min. The dashed line shows phase separation borderline (a) and cream layer thickness (b).

\subsection{Storage stability}

The physical stability of the formulated nanoemulsions was evaluated by monitoring the droplet size distribution and visual observations after one month storage at ambient temperature $\left(25 \pm 4{ }^{\circ} \mathrm{C}\right)$. Any cream layer on top of the samples (stored in tightly sealed glass tubes) was considered as a sign of physical instability.

\subsection{Rheological properties}

A viscometer (DV III ULTRA Brookfield Engineering Laboratories, Stoughton, USA) equipped with concentric cylinders geometries (ULA, SC4-18 and SC4-34) was utilized to measure the flow properties of the low viscosity nanoemulsions at $25^{\circ} \mathrm{C}$. Then, the fitting rate of shear stress $v s$. shear rate data were 

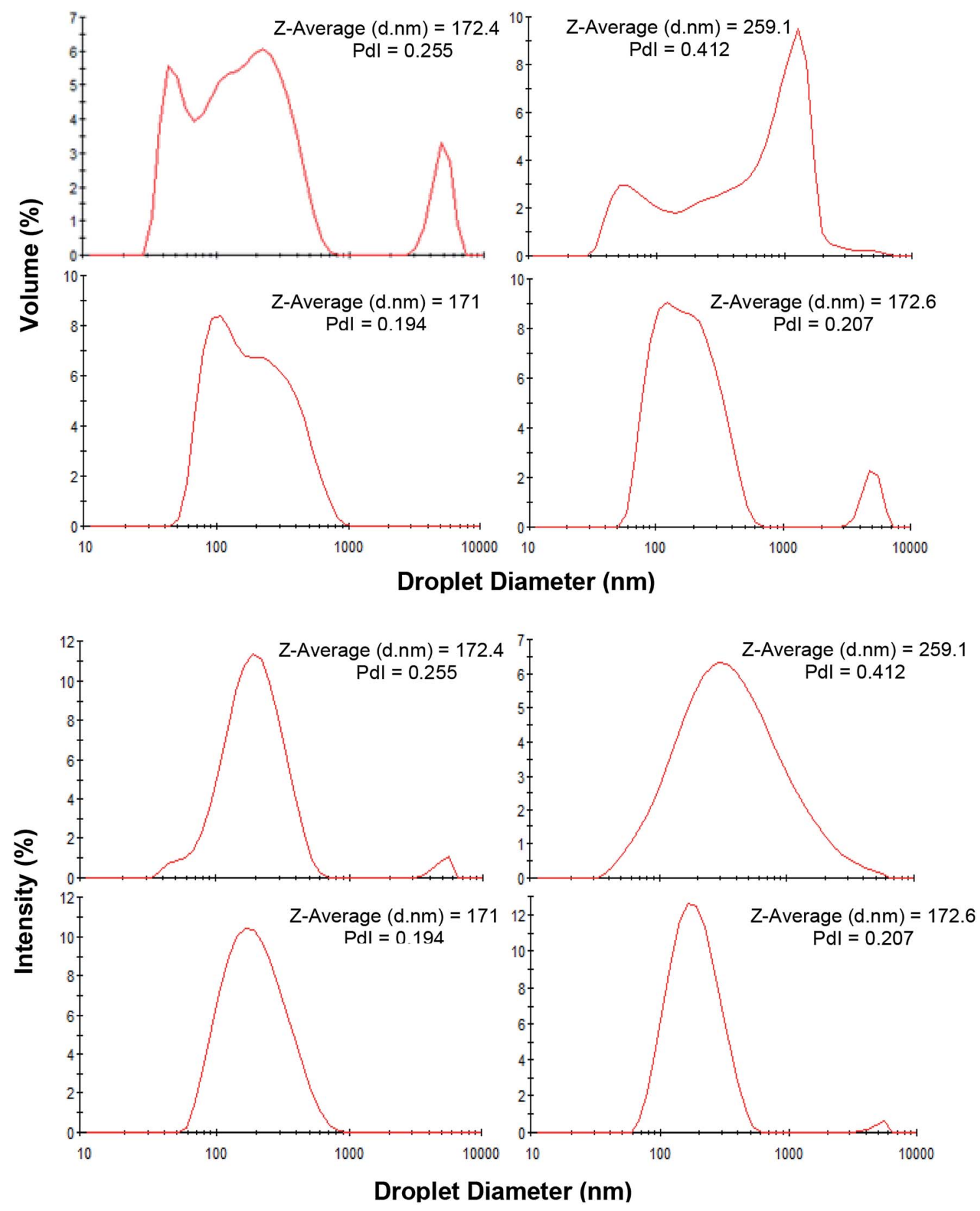

Fig. 3 Droplet size distributions (volume-based and intensity-based) of $10 \mathrm{wt} \%$ freshly prepared (left) and 30 days old (right) nanoemulsions containing 1 (top row) and 2 wt\% (bottom row) saponin prepared under pulsed sonication (9 min, 100\% amplitude).

tested against power law $\left(\tau=k \gamma^{n}\right)$, Herschel-Bulkley $\left(\tau=k \gamma^{n}+\right.$ $\left.\tau_{0}\right)$, Bingham $\left(\tau=k \gamma+\tau_{0}\right)$ and Casson $\left(\sqrt{\tau}=k \sqrt{\gamma}+\sqrt{\tau_{0}}\right)$ models. In these models, $\gamma$ is the shear rate $\left(\mathrm{s}^{-1}\right), \tau$ is the shear stress $(\mathrm{Pa}), k$ is the consistency coefficient $\left(\mathrm{Pa} \mathrm{s}^{n}\right), n$ is the flow behavior index (dimensionless), and $\tau_{0}$ is the yield stress (Pa).

A rheometer (Physica MCR 301, Anton Paar GmbH, Graz, Austria) equipped with a parallel plates geometry (dia $=40 \mathrm{~mm}$, gap size $=1 \mathrm{~mm}$ ) was also utilized to determine the viscoelastic properties. Strain sweep test $(0.1-100 \%)$ performed under a constant frequency $(1 \mathrm{~Hz})$ to determine the linear viscoelastic region (LVR), where rheological properties were independent of stress or strain. Then, a frequency sweep $\left(0.01<\omega<100 \mathrm{rad} \mathrm{s}^{-1}\right)$ was performed within the linear viscoelasticity domain $(0.1 \%)$ for each sample.

\subsection{Statistical analysis}

The preparation of all nanoemulsions and subsequent measurements were carried out in duplicate. Statistical analysis 


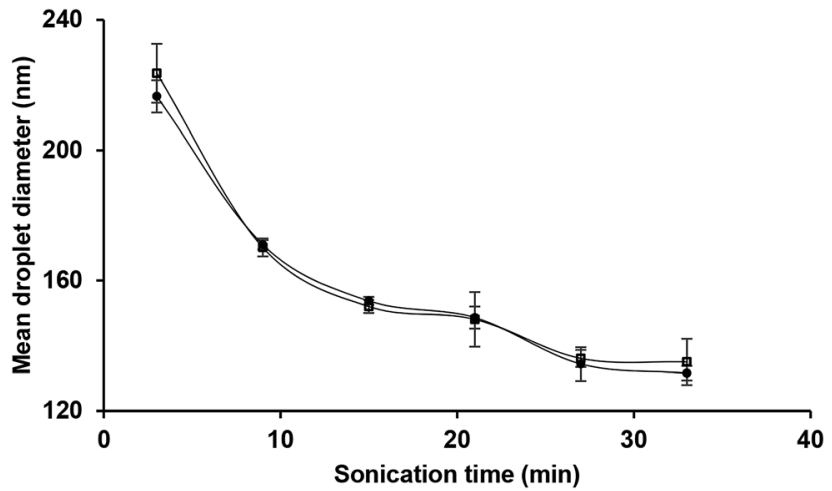

Fig. 4 Effects of sonication time and storage (day $1 \bullet$ and day $30 \square$ ) on the mean droplet diameter of nanoemulsions (10 wt\% sunflower oil, 2 wt\% saponin).

was performed using SPSS (SPSS Inc., Chicago, IL, version 21) and Duncan multiple range test was used to compare mean values at a confidence level of $\alpha<0.05$.

\section{Results and discussion}

\subsection{Effect of emulsifier type and concentration on nanoemulsion formation}

At first, the effect of various concentrations of four emulsifiers on the formation of nanoemulsions under constant oil content (10 wt $\%$ ) and ultrasonication (9 min, amplitude 100\%) was evaluated. With regard to the nanoemulsions which were stabilized by saponin, WPI and lecithin, there was a mean droplet diameter decrease with increasing (from 0.5 to $6 \mathrm{wt} \%$ ) emulsifier concentration (Fig. 1). This was mainly occurred due to the greater decrease in interfacial tension and also high adsorption rate at higher emulsifier levels, which facilitated the droplet breakdown and retarded droplet recoalescence. ${ }^{\mathbf{1 1 , 3 2}}$

As is evident (Fig. 1), two different droplet size regions: (1) the initial steeply decrease and (2) a plateau at lower and higher emulsifier concentrations were discriminated, respectively. Indeed, the reduction trend of droplet diameter followed power law $\left(D=a C^{b}\right)$ model with $R^{2}$ values of $0.67,0.97$ and 0.90 for saponin, WPI and lecithin, respectively. In power law equation, $b$ indicates the rate of decrease and $a$ represents the estimate of droplet diameter $(D)$ at an emulsifier concentration $(C)$ of $1 \mathrm{wt} \%$. The $b$ and $a$ values for saponin $(0.04,180)$, WPI $(0.5,470)$ and lecithin $(1.1,989)$ based (nano)emulsions were wide. Such significant differences among emulsifiers indicate that at lower emulsifier concentration, smaller droplets could be produced using saponin rather than two other emulsifiers. The power law correlation of mean droplet diameter reduction with emulsifier concentration has already been reported for various synthetic (Erramreddy \& Ghosh, 2014) ${ }^{38}$ and natural emulsifiers (Bai and Huan et al., 2016). ${ }^{28}$ These two reports attributed the droplet size plateau to the inefficiency of the emulsification apparatus on sufficient size reduction of droplets.

The minimum emulsifier requirement to create nanoemulsion $(D<200 \mathrm{~nm})$ was $\sim 6$ and $0.5 \mathrm{wt} \%$ for lecithin and saponin with corresponding droplet diameters of 198 and $192 \mathrm{~nm}$, respectively. However, the minimum droplet diameters achieved by WPI ( $6 \mathrm{wt} \%$ ) was $207 \mathrm{~nm}$. As it can be seen (Fig. 2a), all SMP based emulsions were phase separated with a distinct cream layer and a low-opaque bottom phase which did not change when SMP concentration was increased. SMP is a bio-based, non-ionic, low molecular weight, and poor water solubility emulsifier which has already shown great capability in nanoemulsification of lime peel essential oils. ${ }^{33,34}$ However, the nanoemulsification of triacylglycerols, due to inherent physicochemical characteristics such as; low polarity, high interfacial tension, and high viscosity, is often more challenging than flavor oils, essential oils and various lipophilic nutraceuticals. ${ }^{35}$ This is likely the reason for which the SMP was unable of successful emulsification of sunflower oil.

As discussed, saponin, likely owing to its unique physicochemical properties, was more appropriate than other three emulsifiers for the production of nanoemulsions. For instance, its interfacial tension is much smaller than the others: saponin $\left(5 \mathrm{mN} \mathrm{m}^{-1}\right)<$ WPI $\left(9 \mathrm{mN} \mathrm{m}^{-1}\right)<$ lecithin $\left(\sim 20 \mathrm{mN} \mathrm{m}^{-1}\right)<\operatorname{SMP}\left(35 \mathrm{mN} \mathrm{m}^{-1}\right) \cdot{ }^{28,36}$ Consequently, it would be expected to screen the unfavorable thermodynamic interactions at the interface.

Table 1 Comparison of exposure sonication time and oil content effects on the nanoemulsification energy and power density of saponin based nanoemulsions (50 mL, SOR 2 : $10 \mathrm{wt} \%$ )

\begin{tabular}{lcclcl}
\hline Oil content (wt\%) & Sonication time $(\mathrm{min})$ & $\begin{array}{l}\text { Total energy } \\
\text { delivered }(\mathrm{kJ})\end{array}$ & $\begin{array}{l}\text { Energy density } \\
(\mathrm{kJ} \mathrm{mL})\end{array}$ & $\begin{array}{l}\text { Mean droplet diameters } \\
(\mathrm{nm})\end{array}$ \\
\hline & 3 & 26.96 & 0.54 & 3.00 & $216.5 \pm 5.0$ \\
& 9 & 78.57 & 1.57 & 2.91 & $171.0 \pm 1.4$ \\
& 15 & 136.78 & 2.74 & 3.04 & $153.8 \pm 1.2$ \\
& 21 & 183.28 & 3.67 & 2.91 & $148.6 \pm 3.4$ \\
& 27 & 233.42 & 4.67 & 2.88 & $134.3 \pm 5.2$ \\
20 & 33 & 297.03 & 5.94 & 3.00 & $131.5 \pm 2.2$ \\
30 & 15 & 135.19 & 2.70 & 3.00 & $148.4 \pm 0.8$ \\
40 & 15 & 138.60 & 2.77 & 3.01 & $147.0 \pm 1.6$ \\
50 & 15 & 147.27 & 2.94 & 3.27 & $141.3 \pm 10.2$ \\
60 & 15 & 157.48 & 3.15 & 3.50 & $138.5 \pm 9.5$ \\
& 3 & 28.44 & 0.57 & 3.16 & $170.1 \pm 1.6$
\end{tabular}



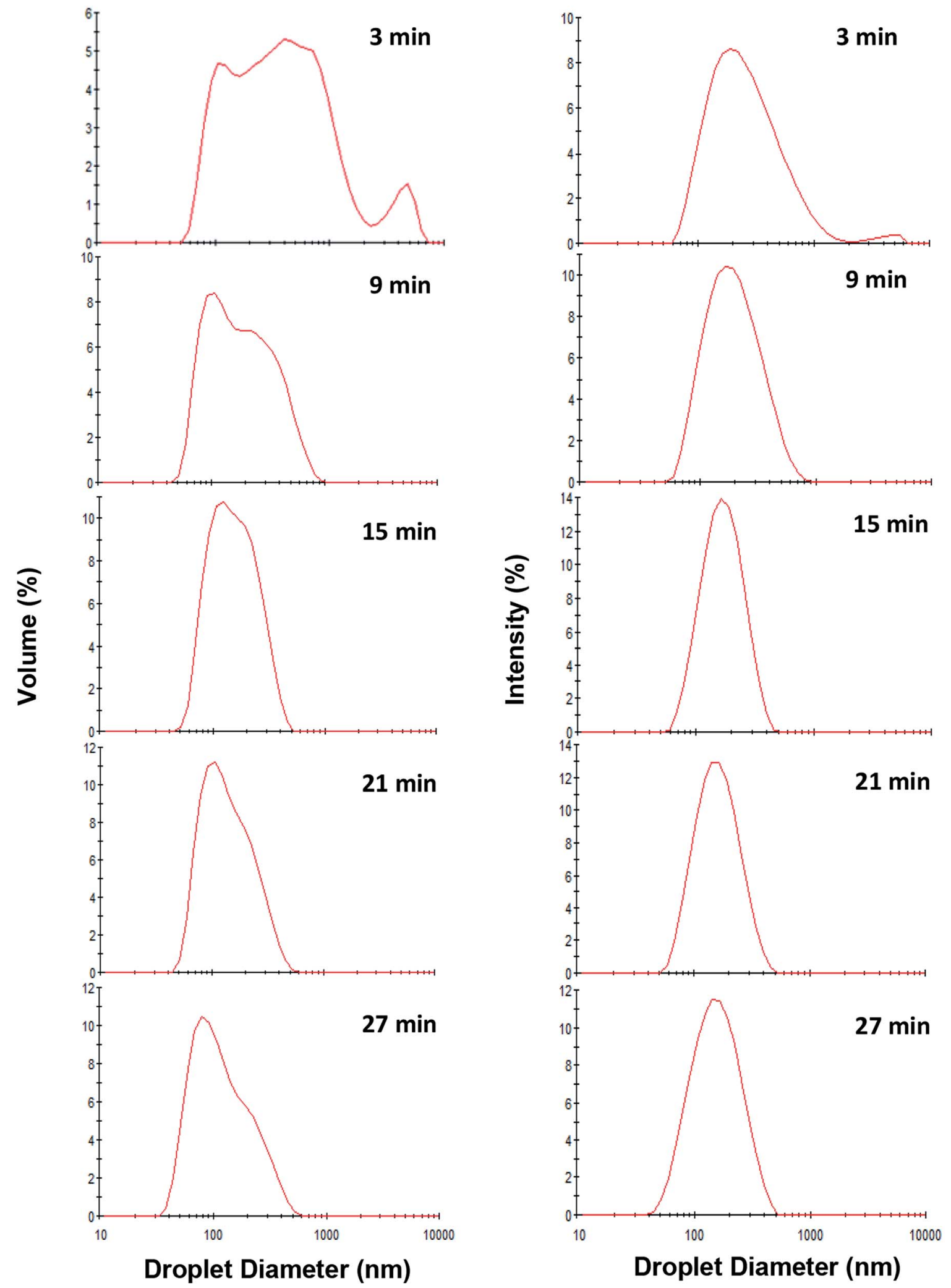

Fig. 5 Effect of exposure to sonication (3, 9, 15, 21 and $27 \mathrm{~min}$ ) on volume-based (Left) and intensity-based (Right) droplet size distribution of fresh oil-in-water nanoemulsions (10 wt\% sunflower oil, 2 wt\% saponin).

The surface load $\left(T, \mathrm{mg} \mathrm{m}^{-2}\right)$ is another useful measure to compare the emulsifying ability of different emulsifiers; the higher the surface load, the greater the amount of emulsifier which is required to cover a given surface area. It $\left(\Gamma=d C_{\text {ads }} / 6 \Phi\right)$ is related to the disperse phase volume fraction $(\Phi)$, mean droplet diameter $(d, \mathrm{~m})$ and concentration of the adsorbed 
a)

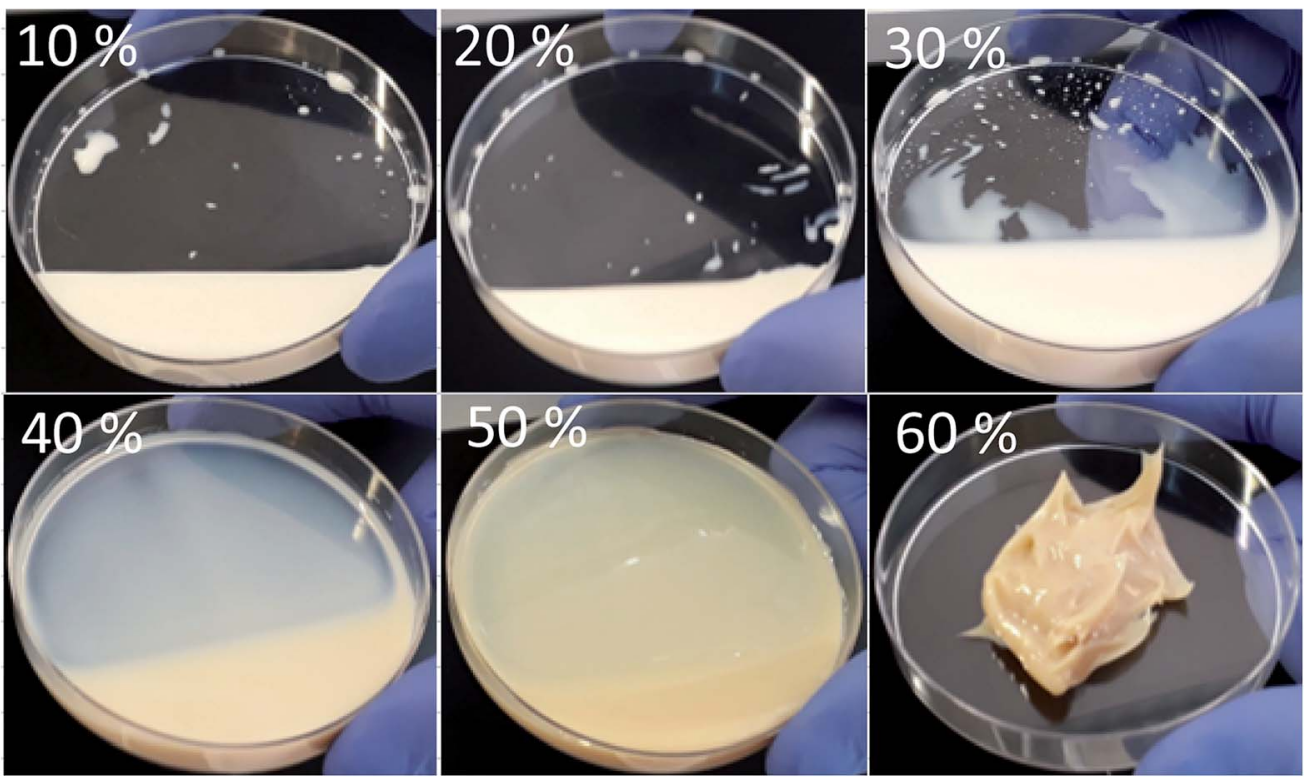

b)

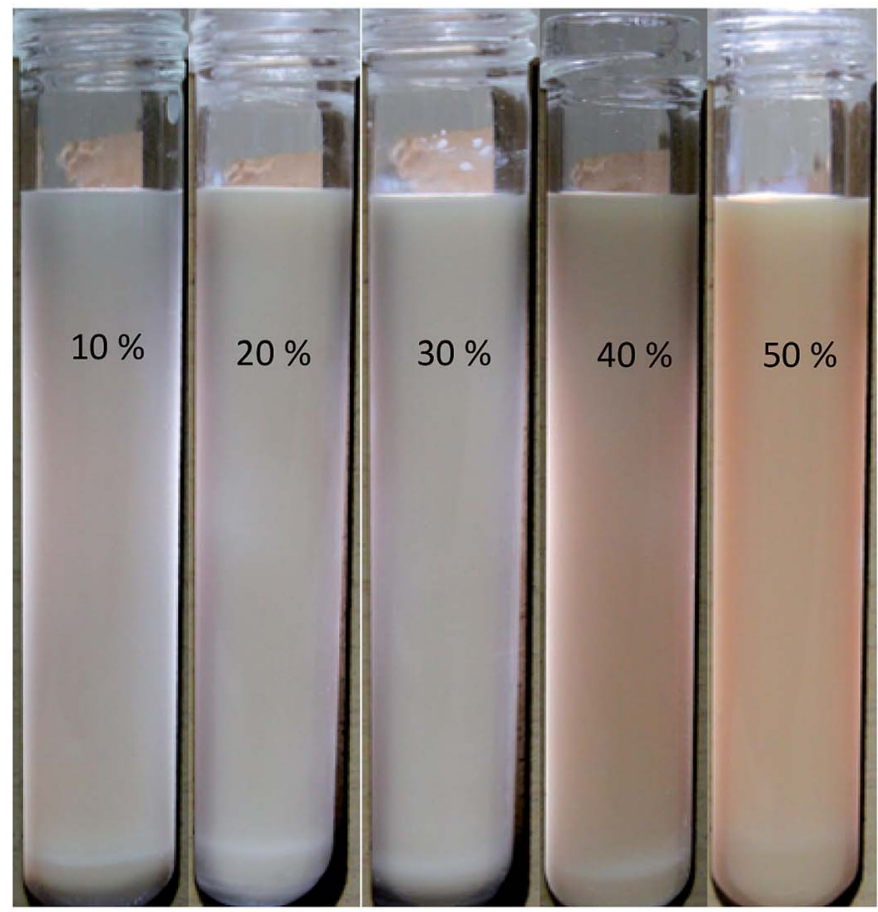

Fig. 6 Comparison of the physical state (a) and visual appearance (b) of saponin based nanoemulsions (10-60 wt\% sunflower oil, SOR 2 : 10 wt\% saponin) after sonication for $15 \mathrm{~min}$ (except one with $60 \%$ which was sonicated for $3 \mathrm{~min}$ ). For better distinction samples were illuminated.

emulsifier when the droplet size reaches a plateau $\left(C_{\text {ads }}, \mathrm{kg}\right.$ $\left.\mathrm{m}^{-3}\right) .{ }^{11}$ Considering $10 \mathrm{wt} \%$ oil phase $(\Phi=0.1)$, the $C_{\text {ads }}=0.5-$ $6 \mathrm{wt} \%\left(5-60 \mathrm{~kg} \mathrm{~m}^{-3}\right)$ and the measured mean droplet diameters, $\Gamma$ for saponin, WPI and lecithin would be $2.87,20.67$ and $19.88 \mathrm{mg} \mathrm{m}^{-2}$, respectively. These numbers suggest that the surface load of saponin is approximately 7 times smaller than WPI and lecithin. Consequently, less saponin is required to cover a given droplet surface area which is highly important from commercial view point as well as health perspective.
Therefore, saponin was selected for further study on manufacturing stable concentrated nanoemulsions and nanogels.

\subsection{Stability of saponin nanoemulsions}

Long-term physical and physicochemical stability are the most valuable advantages of nanoemulsions which could expand their potential applications at commercial products. Therefore, 
the physical appearance, droplet size distribution and zeta potential of saponin nanoemulsions after one month storage under ambient temperature was investigated.

The presence of creaming layer is an easy measure to evaluate the physical instability of the emulsions. Fig. $2 \mathrm{~b}$ demonstrates the nanoemulsions (10 $\mathrm{wt} \%$ oil) with various saponin content (0.5-6 wt $\%)$ which prepared under constant exposure to sonication $(9 \mathrm{~min})$. During visual inspection, a distinct cream layer was observed on all samples though it disappeared upon a gentle mixing. At the presence of lower saponin contents (0.5 and $1 \mathrm{wt} \%$ ), the thickness of cream layer was clearly higher than those with higher levels of emulsifier. It seems that despite the instant nanoemulsion formation at lower saponin level ( $\sim 1 \mathrm{wt} \%$ ), some of the droplets coalesced during storage most likely due to the overpassing of energy barriers and the insufficiency of saponin. These findings are in a good agreement with droplet size measurement after one month storage (Fig. 3). As can be seen, the mean droplet diameter and polydispersity index (PDI) of the saponin nanoemulsions were significantly $(p$ $<0.05$ ) increased after one-month storage.

Interestingly, the nanoemulsions which contained $2 \mathrm{wt} \%$ saponin showed no change in terms of droplet size or PDI though with creaming layer. This suggests that saponin concentration above $2 \mathrm{wt} \%$ was probably higher than what was required for thorough coverage of the nanoemulsion droplets. Therefore, the non-adsorbed excess saponin formed micelles that eventually led to the development of the cream layer through depletion flocculation. Similar effect has already been reported for canola oil and octadecane nanoemulsions which were stabilized by sodium caseinate and SDS, respectively. ${ }^{37,38}$

It needs to be noted that the droplet size distributions of fresh and stored nanoemulsions (sunflower oil $10 \mathrm{wt} \%$, saponin 1 or $2 \mathrm{wt} \%$ ) were also bimodal representing more distinct fractions after storage period (Fig. 3). Interestingly, despite the abundance of saponin ( $2 \mathrm{wt} \%)$, the disrupting ultrasonication energy was not sufficient to form a mono-modal droplet distribution. Consequently, the exposure to sonication was the only option which was required to be manipulated.

\subsection{Effect of sonication time}

Following the findings of the previous sub-section, the influence of sonication time on the mean droplet diameter and polydispersity of the nanoemulsions (containing $10 \mathrm{wt} \%$ sunflower oil and $2 \mathrm{wt} \%$ saponin) was determined (Fig. 4). Overall, there was a profound decrease in mean droplet diameter (from 216 to $131 \mathrm{~nm}$ ) when exposure to sonication was increased (from 3 to $33 \mathrm{~min}$ ). This effect can be attributed to the magnitude of shear stress and disruptive energy generated by acoustic cavitation. As expected (Table 1), by increasing the exposure time to sonication (from 3 to $33 \mathrm{~min}$ ) at a constant oil content (10 wt\%), the total delivered energy was proportionally increased (from 539 to $5941 \mathrm{~J} \mathrm{~mL}^{-1}$ ).

Even after long exposure to sonication (33 min), the resulting droplet size $(131 \mathrm{~nm})$ was still higher than one was reported (Takegami et al., 2008) ${ }^{30}$ on egg yolk phosphatidylcholine nanoemulsions $(50 \mathrm{~nm})$. Such difference could be attributed to its lower oil content ( $5 \mathrm{w} / \mathrm{v} \%$ ), the higher sonication time (60 $\mathrm{min})$, the presence of co-surfactants $(2.2 \mathrm{w} / \mathrm{v} \%$ glycerol and appropriate content of sodium palmitate and sucrose palmitate) and likely the higher emulsifying efficiency of phosphatidylcholine. In another study, on ultrasonic nanoemulsification of Echium plantagineum seed oil ( $5 \mathrm{w} / \mathrm{v} \%$ ), a small mean droplet size $(\sim 225 \mathrm{~nm})$ was achieved by using $20 \mathrm{w} / \mathrm{v} \%$ WPI upon delivering $1750 \mathrm{~J}$ sonication energy ${ }^{39}$ More recently, after long time sonication (40 $\mathrm{min}$ ) using very high concentration of bovine serum albumin (15 wt\%), a corn oil (20 wt\%) coarse emulsion $(525 \mathrm{~nm})$ was formulated ${ }^{25}$ which seems nonsense and non-applicable from commercial view point. Considering the efficiency differences of natural emulsifiers, it can be concluded that by selecting an appropriate emulsifier, surfactant to oil ratio (SOR), and ultrasonication strategy one can formulate a long or medium chain triglyceride nanoemulsion using reasonably reduced quantity of emulsifier, and shorter exposure to sonication without using any co-surfactant.

As is evident (Fig. 4), over the storage period (one month at ambient temperature) there was not any significant $(p>0.05)$ change in the mean droplet diameter of nanoemulsions (10 wt $\%$ sunflower oil and $2 \mathrm{wt} \%$ saponin) prepared under various exposures to sonication. The droplet size distribution of the aged (one month old) nanoemulsions is shown in Fig. 5. With an exception of nanoemulsions which were sonicated for 3, 6 and $9 \mathrm{~min}$, the droplet size distributions were monomodal and the peak was slightly shifted to the left when the sonication time was upturned. In general, monomodal distribution indicates uniformity among droplet sizes and therefore physical stability. Similar monomodal distribution was recently reported for corn oil (10 wt\%) nanoemulsion which was stabilized by saponin (1-10 wt\%, based on an active ingredient basis, i.e., about $14 \mathrm{wt} \%$ of commercial saponin powder) using dualchannel microfluidizer. ${ }^{28}$ In contrast, a bimodal distribution for soybean oil (1-5 wt\%) nanoemulsions that were stabilized by a novel natural emulsifier $(0.5-1.5 \mathrm{wt} \%$ of mucilage extracted from rose cactus leaves or Pereskia aculeate Mill.) under sonication $(15 \mathrm{~min})$ is recently reported. ${ }^{40}$

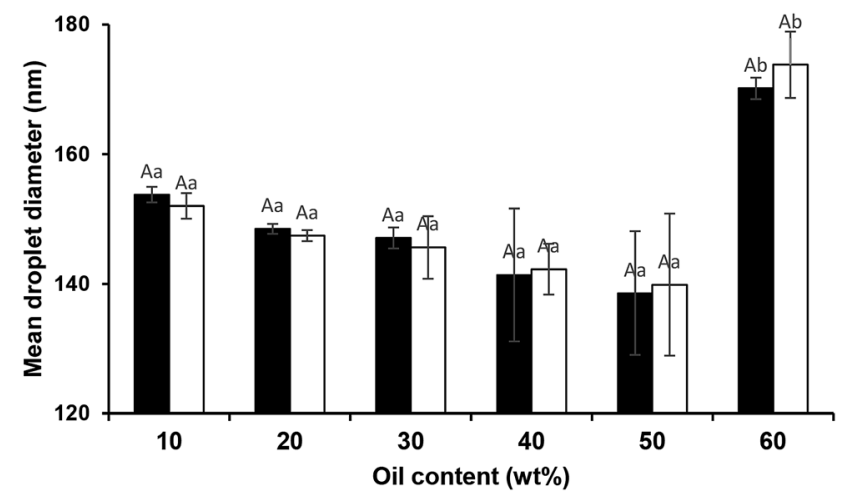

Fig. 7 Influence of oil content and storage (day $1 \square$ and day $30 \mathbf{\square}$ ) on mean droplet diameter of sunflower oil nanoemulsions (SOR 2:10 wt\%). Different small and large letters represent significant differences $(p<0.05)$ amongst oil content and storage, respectively. 


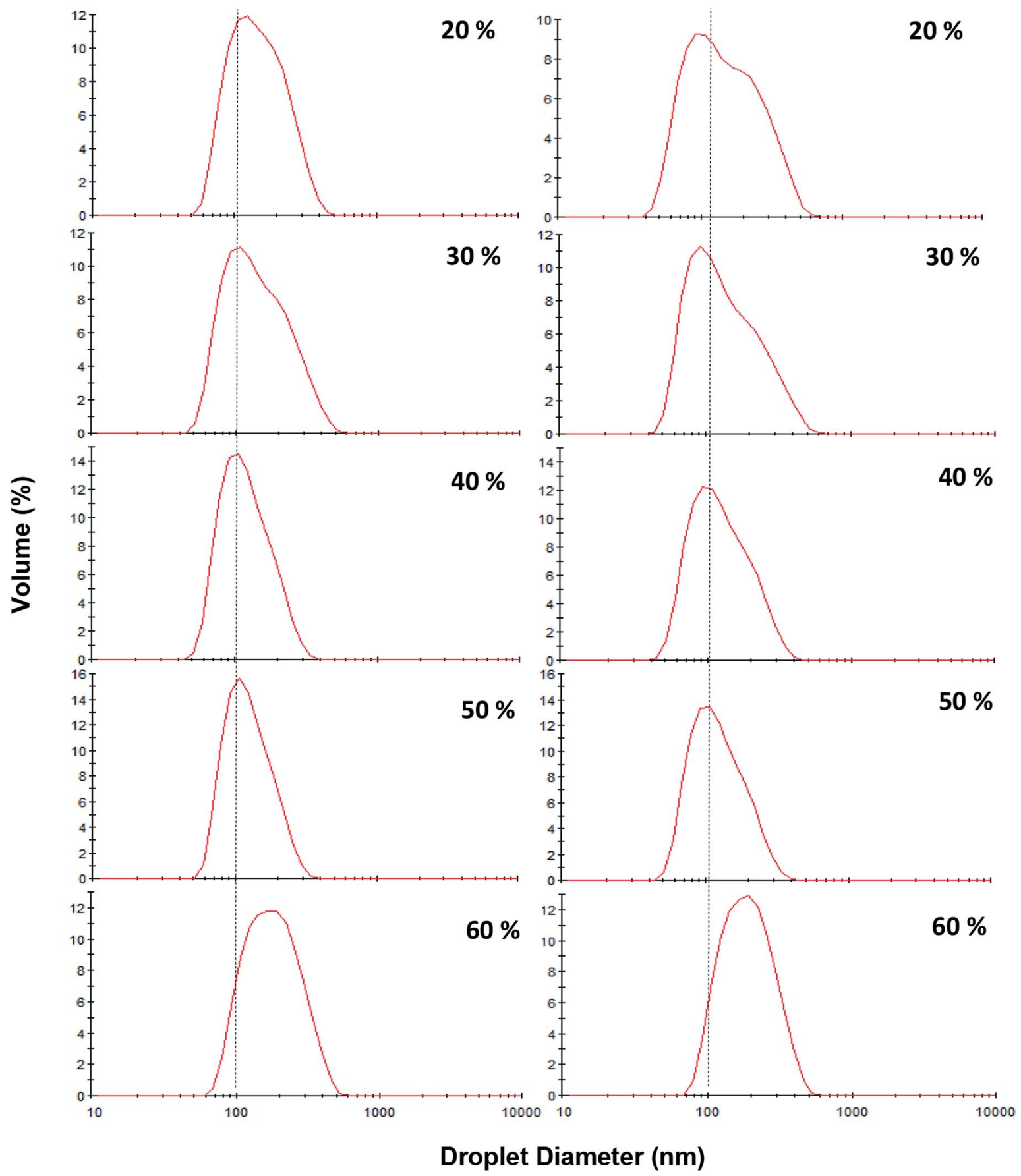

Fig. 8 Influence of oil content (20-60 wt\%) and storage [day 1 (left) and after 30 days storage at ambient temperature (right)] on the droplet size distribution of sunflower oil nanoemulsions (SOR 2 : $10 \mathrm{wt} \%$ ) prepared under pulsed ultrasonication (15 min, 100\% amplitude).

According to the findings of the present study, regarding the droplet size, physical stability and ease of processing, the optimized sonication exposure time (15 $\mathrm{min})$ and SOR $(2: 10)$ were selected for fabrication of the concentrated nanoemulsions at higher levels of oil content (up to $60 \mathrm{wt} \%$ ).

\subsection{Fabrication of concentrated nanoemulsions and nanogels}

In order to investigate the probability of manufacturing concentrated triglyceride nanoemulsions, pre-emulsion mixtures (10-50 wt\% oil at constant SOR $2: 10)$ were sonicated under constant exposure time $(15 \mathrm{~min})$. Then, their creaming and droplet size were monitored over one month storage (Fig. 6). It can be seen that by oil content increase, the viscosity of the nanoemulsions was also increased. Furthermore, at $60 \mathrm{wt} \%$ oil content, a sol-gel transition was occurred albeit after a short exposure to sonication (3 $\mathrm{min})$. The resulting gel was quite rigid and did not flow when the container was tilted. It needs to be noted that sonication was not continued more than 3 min due to its rigidity and unavailability of free 
water to propagate ultrasonic waves as it behaved as a solid or semi-solid object. With regard to the dissipated energy density (Table 1), despite the consistency of exposure to sonication (15 min), it was slightly increased (2736 to $3150 \mathrm{~J} \mathrm{~mL}^{-1}$ ) when oil content was changed (from 10 to $50 \mathrm{wt} \%$ ). This could be likely attributed to the higher viscosity, consequently more resistance against cavitation process. To overcome such a high viscosity, the higher rate of ultrasonic power was required. Although sonication time usually provides a fair assessment of dissipated ultrasound energy, however in the case of concentrated nanoemulsions, the optimization of proportional sonication time and delivered energy should also be considered.

With regard to the mean droplet diameter, it can be seen (Fig. 7) that it was independent of oil content (from 10 to $50 \mathrm{wt} \%$ ). However, some studies ${ }^{25,41}$ reported direct relation between oil content and droplet size under constant sonication conditions. Moreover, the findings of the present study clearly showed that the ultrasound technique alongside natural emulsifiers is highly capable of fabricating edible concentrated nanoemulsions. However, it has been recently ${ }^{28}$ reported that the fabrication of concentrated emulsions ( 40 or $50 \%$ oil) even by dual-channel microfluidization using Quillaja saponins was a great challenge. It has been also shown that the smallest mean droplet diameter $(380 \mathrm{~nm})$ for medium chain triglyceride oil (MCT, $50 \mathrm{wt} \%$ ) emulsion was only achieved when it was homogenized (89.6 $\mathrm{MPa}, 1$ pass) at the presence of $2 \mathrm{wt} \%$ Quillaja saponins plus $1 \mathrm{wt} \%$ lecithin. $^{\mathbf{4 2}}$

During storage at $25{ }^{\circ} \mathrm{C}$, no significant changes in the mean droplet diameter of the nanoemulsions (different oil levels) was observed (Fig. 7). Furthermore, similar to dilute nanoemulsion (10 wt\% oil), the droplet size distributions of the concentrated nanoemulsions (20 to $60 \mathrm{wt} \%$ ) were mono-modal without any creaming or considerable changes over the storage period (Fig. 8). These results proved that saponin was able to stabilize concentrated nanoemulsions and nanogels under appropriate homogenization conditions. It, as a natural emulsifier, belongs to glycosides and is structurally made of two parts; one is hydrophobic which is comprised of quillaic and gypsogenic acids, while its hydrophilic portion is consisted of sugars such as rhamnose, xylose, arabinose, galactose, fucose, and glucuronic acid. ${ }^{43}$ Although it is generally considered a non-ionic surfactant, however it contains a weak dissociating acidic groups (carboxylic groups), originated from glucuronic acid, for that reason it is speculated that similar to anionic surfactants it can induce electrostatic effects under certain $\mathrm{pH}$ and ionic strength. Its relatively high conductivity value $(\sim 600 \mu \mathrm{S} \mathrm{cm})$ against sodium dodecylsulphate $(\sim 1200 \mu \mathrm{S} \mathrm{cm})$ both at $1 \mathrm{wt} \%$ indirectly supports this hypothesis though many other concerns are needed to be addressed. Consequently, it can be easily adsorbed on the droplets (during breakup by sonication) to generate the partly negative charged interfacial coatings or even the protuberance of its hydrophilic parts into the aqueous phase to prevent the recoalescence of droplets through adequate electrostatic and steric repulsions. This speculation was confirmed by measuring the $\zeta$-potentials of the concentrated nanoemulsions where they all were highly negative (about $-60 \mathrm{mV}$ ) at $\mathrm{pH} 4$ and 7 (Table 2). In addition, their particle sizes (in particular 10, 50 and $60 \mathrm{wt} \%$ oil) were relatively constant at both $\mathrm{pH}$ values ( 4 and 7 ). This could be attributed to the $\mathrm{p} K_{\mathrm{a}}$ value (3.18) of glucuronic acid, so that at the pHs above this value (i.e., 4 and 7 in this study), the carboxylic acid groups were partly charged as $-\mathrm{COO}^{-} .^{43}$

\subsection{Rheological properties of nanoemulsions}

Fig. 9a shows the shear stress-shear rate relationship of nanoemulsions (oil content 10-50 wt\%, SOR $2: 10$ ) sonicated for $15 \mathrm{~min}$. Based on the highest determination coefficient $\left(R^{2}\right)$ and the lowest root mean standard error (RMSE), almost all nanoemulsions fitted well (Table 3$)$ with the power law $\left(\tau=K \gamma^{n}\right)$ model. As mentioned earlier, owing to the gelation of the highly concentrated one (60 wt\% oil) its flow properties was not measured (Fig. 6a).

Resembling the flow behavior, the apparent viscosity versus shear rate curves of the nanoemulsions with different oil concentrations (Fig. 9b, 1-3) also confirmed that at lower oil concentrations $(10,20$ and $30 \mathrm{wt} \%)$, especially at higher shear rate (about $150 \mathrm{~s}^{-1}$ ), the apparent viscosity was almost independent of shear rate and their flow behavior indices $n(2.347$, 1.042 and 1.023) indicated non-pseudoplastic behavior (Table 3). However, at the higher oil concentrations (40 and $50 \mathrm{wt} \%$ ), with increasing shear rate, apparent viscosity considerably decreased and their flow behavior resembled pseudoplastic

Table 2 Comparison of the influence of oil content and pH on the mean droplet size and zeta potential of nanoemulsions stabilized by 15 min sonication using saponin (SOR 2 : $10 \mathrm{wt} \%$ )

\begin{tabular}{|c|c|c|c|c|}
\hline Oil content (wt\%) & $\mathrm{pH}$ & $\begin{array}{l}\text { Mean droplet diameters } \\
\text { after } 1 \text { day }(\mathrm{nm})\end{array}$ & Zeta potential $(\mathrm{mV})$ & $\begin{array}{l}\text { Mean droplet diameters } \\
\text { after } 30 \text { days (nm) }\end{array}$ \\
\hline 10 & 4 & $146.6 \pm 7.3$ & -54.0 & $146.3 \pm 1.4$ \\
\hline 20 & 7 & $148.4 \pm 0.8$ & -63.0 & $147.4 \pm 0.8$ \\
\hline 30 & 7 & $147.0 \pm 1.6$ & -62.5 & $145.46 \pm 4.8$ \\
\hline 40 & 7 & $141.3 \pm 10.2$ & -59.8 & $142.2 \pm 3.9$ \\
\hline \multirow[t]{2}{*}{60} & 4 & $186.7 \pm 6.8$ & -49.1 & $187.6 \pm 1.1$ \\
\hline & 7 & $170.1 \pm 1.6$ & -66.7 & $173.8 \pm 5.1$ \\
\hline
\end{tabular}



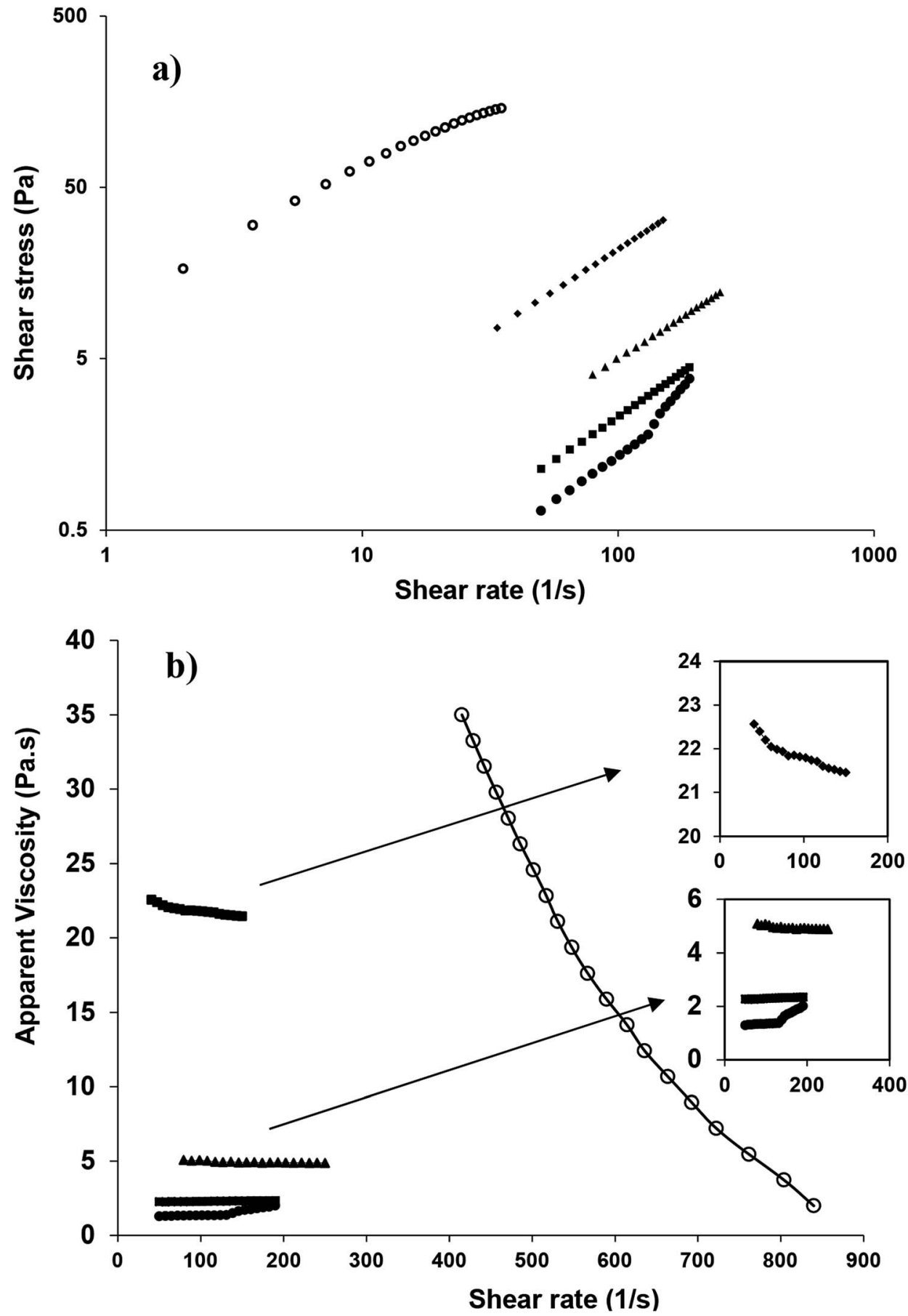

Fig. 9 Changes in shear stress (a) and apparent viscosity (b) of sunflower oil nanoemulsion as a function of shear rate [SOR, 2 : $10 \mathrm{wt} \%, 10$

(shear thinning) nature with smaller flow indices (0.965 and 0.666). Such high dependency of flow behavior with the oil concentration (volume fraction) had been previously reported for nanoemulsions that were stabilized by synthetic and natural emulsifiers. $^{\mathbf{3 8 , 4 0 , 4 1}}$ However, the consistency values $(k)$ were much higher for nano/submicron emulsions ( $<10 \mathrm{wt} \%$ oil) which were prepared under sonication by WPI and fenugreek gum, ${ }^{41}$ or mucilage extracted from the cactus leaves ${ }^{\mathbf{4 0}}$ than those for the present study ( $<40 \mathrm{wt} \%$ oil). Such dissimilarity could be attributed to the predominant viscous contribution of those gums and/or emulsifiers on the pseudoplasticity of the emulsions. In addition, when the oil concentration was increased (i.e., from 40 to $50 \mathrm{wt} \%$ ), the apparent viscosity abruptly (almost 19 times) changed (i.e., from 22 to $415 \mathrm{~Pa} \mathrm{~s}$ at $40 \mathrm{~s}^{-1}$ ). The low viscosity of the former one, despite its relatively high oil level, could have a potential importance in manufacturing oil-soluble drug loaded emulsions for instance for parenteral nutrition 
Table 3 Flow behavior of saponin based nanoemulsions (SOR 2 : $10 \mathrm{wt} \%, 15$ min sonication) fitted to rheological models

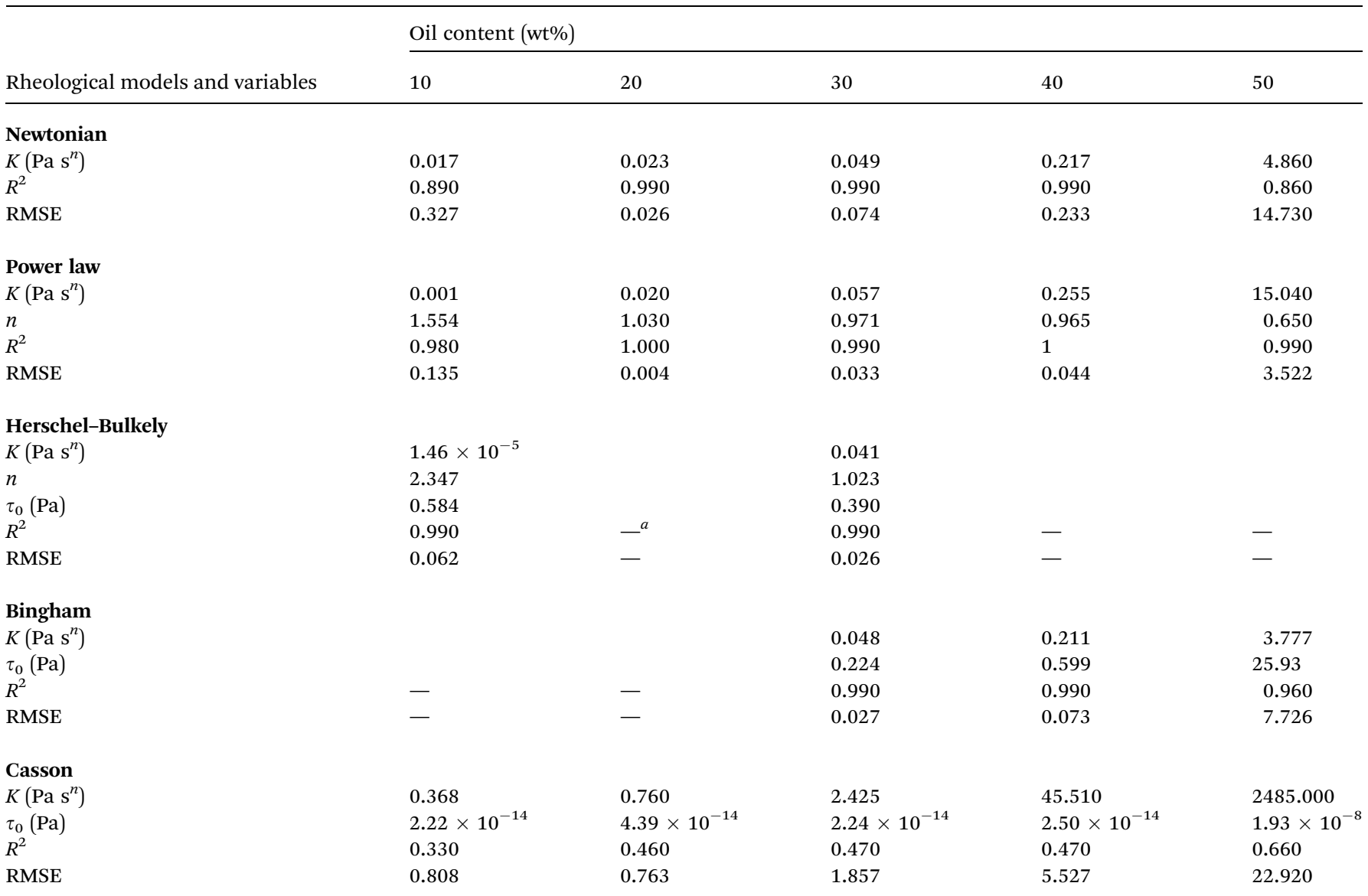

${ }^{a}$ Data fitting to Herschel-Bulkely and Bingham models for these samples indicated negative values of yield stress, so the obtained results for these two models in such samples were not reliable.

purposes, as it could facilitate its injectibility through narrow needles.

Fig. 10a depicts the strain dependencies of storage $\left(G^{\prime}\right)$ and loss $\left(G^{\prime \prime}\right)$ moduli for saponin-stabilized nanoemulsions containing 40,50, and $60 \mathrm{wt} \%$ oil which were sonicated for $15 \mathrm{~min}$. It can be seen that for all three nanoemulsions, $G^{\prime}$ was significantly greater than $G^{\prime \prime}$, up to a certain strain, indicating their dominant elastic nature. However, the first two cases flowed under gravity (Fig. 6a), demonstrating weak gel. This was also reflected in dynamic rheological parameters (Table 4). The two former concentrated nanoemulsions (40 and $50 \mathrm{wt} \%$ oil) had the lowest values of $\gamma_{\mathrm{L}}$, i.e., the limiting value of the linear viscoelastic range (Table 4), while its $\gamma_{\mathrm{L}}$ value was larger $(1.54 \%)$ for the one with $60 \mathrm{wt} \%$ oil. These findings indicate that this sample had longer linear viscoelastic range (LVR), consequently higher stability under the $\gamma$-amplitude. Moreover, the highest $\tau_{y}$ and $G_{\mathrm{LVR}}^{\prime}$ belonged to the one with $60 \mathrm{wt} \%$ oil manifesting its higher mechanical force requirement to flow and more rigid structure, respectively (Table 4).

The solid-like behavior of the latter nanoemulsion $\left(G^{\prime} \gg G^{\prime \prime}\right)$ was also confirmed by frequency sweep (0.1-126 $\left.\mathrm{rad} \mathrm{s}^{-1}\right)$ test (Fig. 10b). To model the frequency dependency of $G^{\prime}$, the power law model $\left(G^{\prime}=a \omega^{b}\right)$ was fitted to frequency sweep data and resulting parameters are presented in Table $4 . a$ is the magnitude of $G^{\prime}$ at a given $\omega(1 \mathrm{~Hz})$ and denotes the strength of the interactions between rheological units. $b$ is also an indication of relative viscoelasticity and can be determined from the slope of $G^{\prime}$ versus $\omega$ in a log-log plot. Indeed, the $b$-value indicates the extent of interaction or the number of rheological units entangled with one another in a three dimensional structure. It is already reported that for the viscous, elastic, and covalent gels, $b$ should be $\approx 1,<1$, and $=0$, respectively. ${ }^{44}$ Considering such criteria, the concentrated nanoemulsion containing 40,50 and $60 \mathrm{wt} \%$ oil, owing to their smaller $b$ values $(<1)$ could be presumed to be elastic gels but with different strength $(a \simeq$ 3909 and 5434 and 31155 Pa s, respectively).

The concentrated nanoemulsions stabilized by saponin, in the current study, revealed notably weaker rheological properties than those with SDS. ${ }^{12}$ For instance, the nanoemulsions (50 or $60 \mathrm{wt} \%$ oil) which contained SDS converted into non-fluid viscoelastic gels once sonicated for 9 and $3 \mathrm{~min}$, respectively. In addition, the apparent viscosity and dynamic rheological parameters of those with saponin was lower than one with SDS. It is already reported that owing to droplet size reduction and enhancement of the repulsive interactions (the formation of the emulsifier layer at the droplet surface), the effective volume 

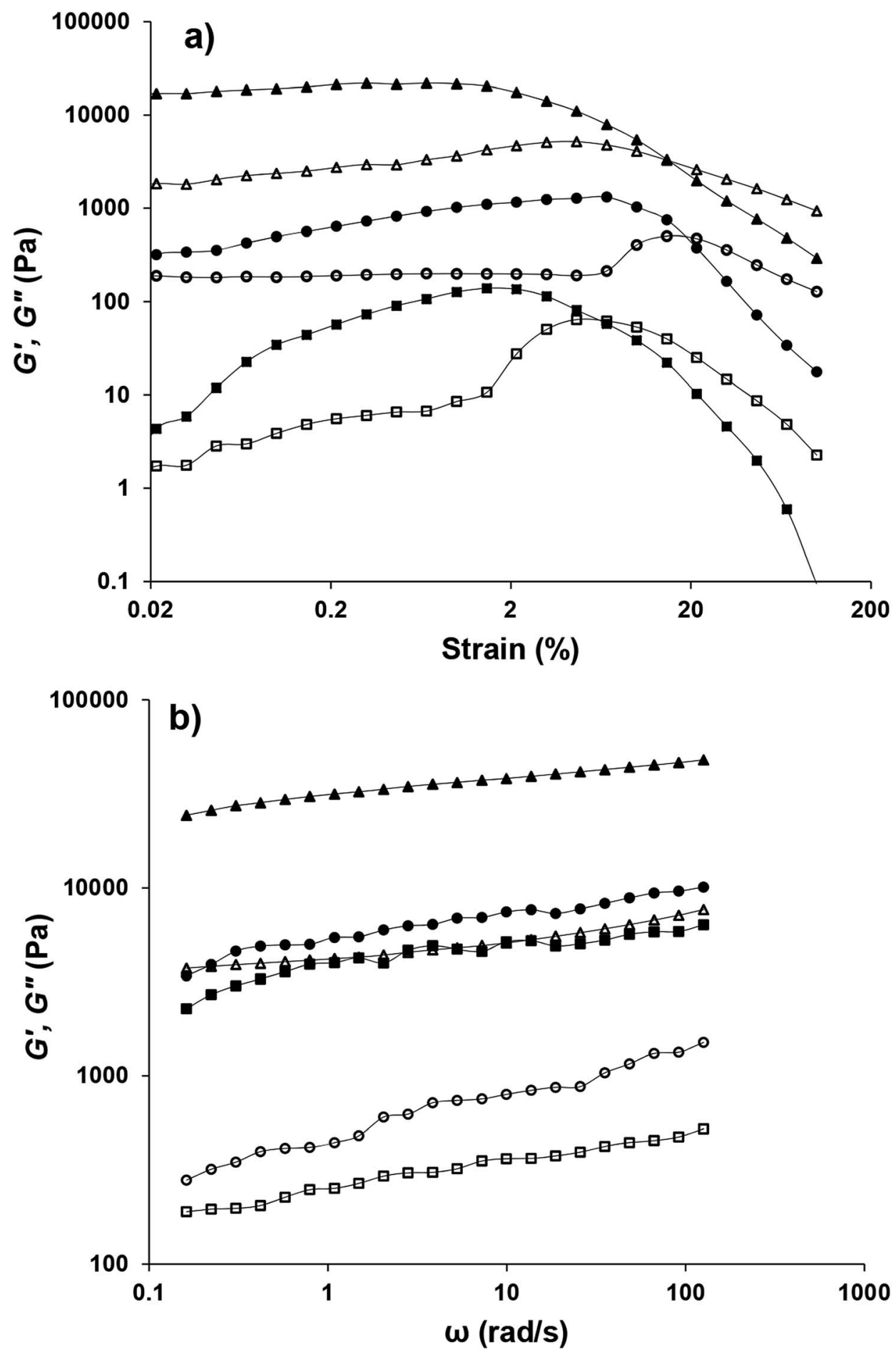

Fig. 10 Comparison of the dependency of $G^{\prime}$ (filled) and $G^{\prime \prime}$ (hollow) on (a) strain and (b) frequency for nanoemulsions containing 40 ( $\square$, $\mathbf{\square}$ ), 50 $(O, \mathbf{O})$ and $60(\triangle, \mathbf{\Lambda})$ wt\% sunflower oil sonicated for 15,15 and $3 \mathrm{~min}$, respectively (SOR 2 : $10 \mathrm{wt} \%$ ).

fraction and rheological properties could change. ${ }^{\mathbf{1 0}}$ Interestingly, there was not a significant difference regarding the mean droplet diameter of $50(138.5 \pm 9.5 \mathrm{~nm}$ against $146.1 \pm 7.2 \mathrm{~nm})$ and $60(170.1 \pm 1.6 \mathrm{~nm}$ against $172.7 \mathrm{~nm}) \mathrm{wt} \%$ nanoemulsions which were stabilized by saponin and SDS, respectively. Hence, there could be other factors than volume fraction and droplet size which likely affect the gelation threshold of nanoemulsions. For instance, the surface potential of the droplets which was significantly high for SDS nanoemulsions $(-84 \mathrm{mV})$ compared to saponin based one $(-60$ to $-66 \mathrm{mV})$. It needs to be emphasized that the purity level of the tested saponin is not known and it is believed this could be a critical factor. Therefore, it can be speculated that such difference can subsequently increase the shell thickness through extending 
Table 4 Influence of the oil content (40,50, and $60 \mathrm{wt} \%$ ) on rheological properties (strain and frequency sweep parameters) of saponin based nanoemulsions (SOR 2 : $10 \mathrm{wt} \%$, sonication time $15 \mathrm{~min}$, except the last row which was sonicated for $3 \mathrm{~min}$ ) ${ }^{a}$

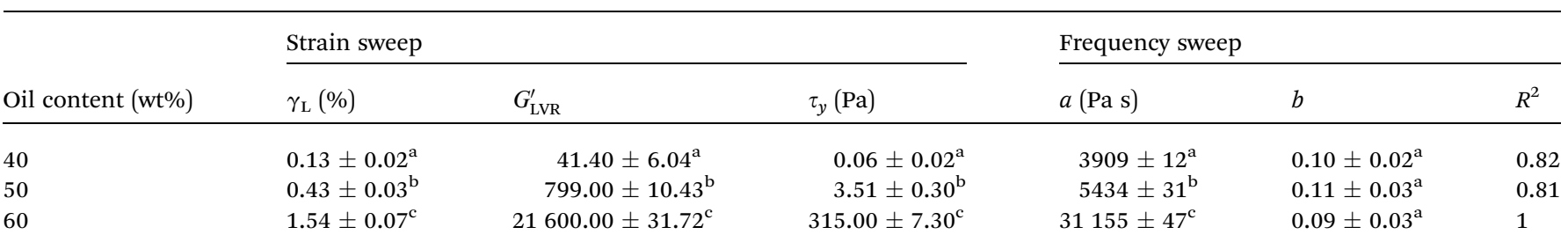

${ }^{a}$ Various small letters at each column represent significant difference $(p<0.05)$.

electric potential into the surrounding aqueous phase. Therefore, by greater overlapping of the electrostatic shells surrounding the droplets, the effective volume fraction and eventually the shear modulus of the nanoemulsions increase.

Apart from some dissimilarities, both surfactants at various SOR $(0.7: 10$ and $2: 10)$ but under $60 \mathrm{wt} \%$ sunflower oil were capable of forming viscoelastic nanogels due to the proximity of the dispersed phase volume fraction to maximal random jamming $(\Phi=0.64)$ level. In such a packed condition, each droplet is entrapped in a network of neighboring droplets. Nevertheless, the detailed mechanisms by which these two emulsifiers differently influence the rheological properties of the concentrated nanoemulsions are needed to be investigated.

In a most recent study, ${ }^{45}$ gelation only observed in $\mathrm{O} / \mathrm{W}$ nanoemulsion (canola oil content $40 \mathrm{wt} \%$ ) which was stabilized by $5 \mathrm{wt} \%$ sodium caseinate but not with WPI likely due to higher shell layer thickness of sodium caseinate resulted from the combined repulsive steric barrier and charge cloud. Similarly in the present study, the possible reason for different nanogelation capability of saponin and SDS could be attributed to the enhancement of the effective volume fraction and the abovementioned mechanisms. It seems the nanogelation capability of saponin, which was utilized used in the present study, was higher than WPI, but lower than sodium caseinate. Furthermore, it was found that the formation of the saponin "skin" on the surface of droplets and subsequent wrinkling after large surface shrinkage can also significantly contribute to the viscoelasticity of emulsions. ${ }^{46}$ This was suggested as a possible explanation for the higher apparent viscosity of the $50 \mathrm{wt} \%$ MCT emulsion prepared with Quillaja saponins (0.5$3 \mathrm{wt} \%)$ compared to the emulsion stabilized by rhamnolipids. ${ }^{7}$

\section{Conclusions}

The current study confirmed the efficacy of saponin as a natural emulsifier in fabricating diluted (10 wt $\%$ oil) and concentrated (20-50 wt\% oil) nanoemulsions and nanogel (60 wt\% oil) using ultrasonication and long chain triglyceride. The macroscopic, droplet size distribution and rheological findings proved the mono-modal droplet size distribution with no change during storage. Despite the majority of the previous reports, no cosurfactant was added and it seems by using pure saponin the SOR could be tremendously reduced. These results could be potentially applicable in development of nanoemulsion applications and nanogels in "label-friendly" soft foods, cosmetic and pharmaceutical products.

\section{Conflicts of interest}

The authors declare that they have no conflict of interests.

\section{Acknowledgements}

The funding supports from the Iran National Science Foundation (INSF\# 95817511, Feb., 28th, 2016) and Tarbiat Modares University (Research and Technology Deputy) are gratefully acknowledged.

\section{References}

1 M. Artiga-Artigas, Y. Lanjari-Pérez and O. Martín-Belloso, Food Chem., 2018, 266, 466-474.

2 R. Kumar, K. Kaur, S. K. Pandey, R. Kumar, S. Uppal and S. K. Mehta, J. Mol. Liq., 2018, 263, 324-333.

3 M. Kaci, A. Belhaffef, S. Meziane, G. Dostert, P. Menu, É. Velot, S. Desobry and E. Arab-Tehrany, Colloids Surf., B, 2018, 167, 165-175.

4 D. J. McClements, Soft Matter, 2011, 7, 2297-2316.

5 A. Gupta, H. B. Eral, T. A. Hatton and P. S. Doyle, Soft Matter, 2016, 12, 2826-2841.

6 R. H. Müller, D. Harden and C. M. Keck, Drug Dev. Ind. Pharm., 2012, 38, 420-430.

7 Z. Li, L. Dai, D. Wang, L. Mao and Y. Gao, J. Agric. Food Chem., 2018, 66, 3922-3929.

8 V. V. Erramreddy and S. Ghosh, Colloids Surf., A, 2015, 484, 144-152.

9 J. N. Wilking and T. G. Mason, Phys. Rev. E, 2007, 75, 041407.

10 Z. Zhang and D. J. McClements, in Nanoemulsions, ed. S. M. Jafari and D. J. McClements, Academic Press, 2018, pp. 2149, DOI: 10.1016/B978-0-12-811838-2.00002-3.

11 D. J. McClements, Food emulsions: principles, practices, and techniques, CRC press, Boca Raton, 3rd edn, 2015.

12 M. Nejatian, S. Abbasi and R. Kadkhodaee, Langmuir, 2018, 34, 11433-11441.

13 T. S. H. Leong, T. J. Wooster, S. E. Kentish and M. Ashokkumar, Ultrason. Sonochem., 2009, 16, 721-727.

14 T. Mehmood, A. Ahmad, A. Ahmed and Z. Ahmed, Food Chem., 2017, 229, 790-796. 
15 M. Wulff-Pérez, A. Torcello-Gómez, M. J. Gálvez-Ruíz and A. Martín-Rodríguez, Food Hydrocolloids, 2009, 23, 10961102.

16 A. Amani, P. York, H. Chrystyn and B. J. Clark, Pharm. Res., 2009, 27, 37-45.

17 A. Mirmajidi-Hashtjin and S. Abbasi, Food Hydrocolloids, 2015, 44, 40-48.

18 J. Carpenter and V. K. Saharan, Ultrason. Sonochem., 2017, 35, 422-430.

19 K. Bhargava, D. S. Conti, S. R. P. da Rocha and Y. Zhang, Food Microbiol., 2015, 47, 69-73.

20 T. P. Sari, B. Mann, R. Kumar, R. R. B. Singh, R. Sharma, M. Bhardwaj and S. Athira, Food Hydrocolloids, 2015, 43, 540-546.

21 V. Ghosh, A. Mukherjee and N. Chandrasekaran, Colloids Surf., B, 2014, 114, 392-397.

22 S. Abbas, K. Hayat, E. Karangwa, M. Bashari and X. Zhang, Food Eng. Rev., 2013, 5, 139-157.

23 S. Kentish, T. J. Wooster, M. Ashokkumar, S. Balachandran, R. Mawson and L. Simons, Innovative Food Sci. Emerging Technol., 2008, 9, 170-175.

24 A. Ali, G. Mekhloufi, N. Huang and F. Agnely, Int. J. Pharm., 2016, 500, 291-304.

25 M. Tabibiazar, S. Davaran, M. Hashemi, A. Homayonirad, F. Rasoulzadeh, H. Hamishehkar and M. A. Mohammadifar, Food Hydrocolloids, 2015, 44, 220-228. 26 L. Alfaro, D. Hayes, C. Boeneke, Z. Xu, D. Bankston, P. J. Bechtel and S. Sathivel, LWT-Food Sci. Technol., 2015, 62, 1184-1191.

27 S. Calligaris, S. Plazzotta, F. Valoppi and M. Anese, Food Res. Int., 2018, 107, 700-707.

28 L. Bai, S. Huan, J. Gu and D. J. McClements, Food Hydrocolloids, 2016, 61, 703-711.

29 S. Abbas, M. Bashari, W. Akhtar, W. W. Li and X. Zhang, Ultrason. Sonochem., 2014, 21, 1265-1274.

30 S. Takegami, K. Kitamura, H. Kawada, Y. Matsumoto, T. Kitade, H. Ishida and C. Nagata, Chem. Pharm. Bull, 2008, 56, 1097-1102.
31 L. Bai and D. J. McClements, J. Colloid Interface Sci., 2016, 479, 71-79.

32 S. Tcholakova, N. D. Denkov and T. Danner, Langmuir, 2004, 20, 7444-7458.

33 J. Rao and D. J. McClements, Food Hydrocolloids, 2011, 25, 1413-1423.

34 E. Sadeghi Namazi and S. Abbasi, presented in part at the 3 th great conference of food science and technology, Isfahan, Iran, 2015.

35 D. J. McClements and J. Rao, Crit. Rev. Food Sci. Nutr., 2011, 51, 285-330.

36 G. Garofalakis, B. S. Murray and D. B. Sarney, J. Colloid Interface Sci., 2000, 229, 391-398.

37 S. Uluata, E. A. Decker and D. J. McClements, Food Biophysics, 2016, 11, 52-59.

38 V. V. Erramreddy and S. Ghosh, Langmuir, 2014, 30, 1106211074.

39 K. Chalothorn and W. Warisnoicharoen, Am. J. Food Technol., 2012, 7, 532-541.

40 A. M. T. Lago, I. C. O. Neves, N. L. Oliveira, D. A. Botrel, L. A. Minim and J. V. de Resende, Ultrason. Sonochem., 2019, 50, 339-353.

41 O. Kaltsa, N. Spiliopoulou, S. Yanniotis and I. Mandala, Food Hydrocolloids, 2016, 61, 625-632.

42 X. Luo, Y. Zhou, L. Bai, F. Liu, R. Zhang, Z. Zhang, B. Zheng, Y. Deng and D. J. McClements, Food Res. Int., 2017, 96, 103112.

43 S. Mitra and S. R. Dungan, J. Agric. Food Chem., 1997, 45, 1587-1595.

44 M. Nejatian, M. Hatami and M. A. Mohammadifar, Int. J. Biol. Macromol., 2013, 53, 168-176.

45 A. D. Patel, M. Sc. thesis, University of Saskatchewan, Canada, 2017.

46 K. Golemanov, S. Tcholakova, N. Denkov, E. Pelan and S. D. Stoyanov, Soft Matter, 2014, 10, 7034-7044. 